\title{
Equidistant map projections of a triaxial ellipsoid with the use of reduced coordinates
}

\author{
Pawel Pędzich \\ Warsaw University of Technology \\ Faculty of Geodesy and Cartography \\ 1 Pl. Politechniki, 00-661 Warsaw, Poland \\ e-mail: ppedzich@wp.pl \\ https://orcid.org/0000-0001-5292-4887
}

Received: 07 September 2017 / Accepted: 26 September 2017

\begin{abstract}
The paper presents a new method of constructing equidistant map projections of a triaxial ellipsoid as a function of reduced coordinates. Equations for $x$ and $y$ coordinates are expressed with the use of the normal elliptic integral of the second kind and Jacobian elliptic functions. This solution allows to use common known and widely described in literature methods of solving such integrals and functions. The main advantage of this method is the fact that the calculations of $x$ and $y$ coordinates are practically based on a single algorithm that is required to solve the elliptic integral of the second kind. Equations are provided for three types of map projections: cylindrical, azimuthal and pseudocylindrical. These types of projections are often used in planetary cartography for presentation of entire and polar regions of extraterrestrial objects. The paper also contains equations for the calculation of the length of a meridian and a parallel of a triaxial ellipsoid in reduced coordinates. Moreover, graticules of three coordinates systems (planetographic, planetocentric and reduced) in developed map projections are presented. The basic properties of developed map projections are also described. The obtained map projections may be applied in planetary cartography in order to create maps of extraterrestrial objects.
\end{abstract}

Keywords: map projection, triaxial ellipsoid, reduced coordinates, geocentric coordinates, planetocentric coordinates

\section{Introduction}

A triaxial ellipsoid is often used as a reference surface in map projections of irregular extraterrestrial objects. Subject literature provides numerous examples of such map projections. Bugayevsky $(1987,1991)$ developed the conformal cylindrical projection of a triaxial ellipsoid. Snyder (1985) presented the triaxial equivalent of the Mercator projection. Different versions of conformal projections of a triaxial ellipsoid were created by Nyrtsov (2014), who developed such a projection with use of elliptical 
coordinates. Fleis et al. (2013) presented a conformal projection that maintained the angle between parallels and meridians. Nyrtsov at al. (2013) presented an equalarea map projection of a triaxial ellipsoid. Bugaevsky (1999) created a cylindrical equidistant along meridians map projection of a triaxial ellipsoid. The equations used to calculate the lengths of meridians were in a form of trigonometric series. Nyrtsov at al. (2012) also presented cylindrical and azimuthal equidistant along meridians map projections of a triaxial ellipsoid. Equations for calculating the lengths of meridians were in a form of elliptic integrals (not in the canonical forms) and the method of numerical integration was proposed.

Planetocentric and planetographic coordinates are often used to describe a triaxial ellipsoid and their map projections (Fleis et al., 2013; Nyrtsov at al., 2012; Nyrtsov, 2013; 2014). In literature, the term "geocentric" is sometimes used instead of planetocentric and geodetic or geographic instead of planetographic coordinates (Bektaş, 2014; 2015). However, such term with the "geo-" prefix should refer only to the terrestrial ellipsoid. As far as the ellipsoids of extraterrestrial objects are concerned, the "planeto-" prefix should be used instead of "geo-". Equations for a triaxial ellipsoid in planetographic coordinates may be found in (Nyrtsov at al., 2007) and with the use of planetocentric coordinates in (Nyrtsov, 2015). These equations enable to calculate 3D Cartesian coordinates on the basis of planetocentric and planetographic coordinates. Nyrtsov (2012) provided the definitions of both coordinates systems. Bektaş (2015) in turn presented the definition of geodetic coordinates on a triaxial ellipsoid, which is convergent with Nyrtsov's (2012) definition of planetographic coordinates. He also presented the differences between graticules in geodetic coordinate systems on a triaxial and on an oblated ellipsoid. Planetocentric coordinates are often used in sets of data, e.g. data of surface models of extraterrestrial objects, available on NASA website (sbn.psi.edu/pds/archive/sat.html). Also on the IAU (International Astronomical Union) website (planetarynames.wr.usgs.gov) there is a gazetteer of planetary nomenclature with sets of data with planetocentric coordinates. On the other hand, the heights of points are determined from the surface along normal vector. Hence planetographic coordinates are important to create maps of extraterrestrial objects, too. As the descriptions of planetographic and planetocentric coordinate systems in literature are sufficient, the author decided not to publish their characteristics. Readers can find more information about coordinate systems of a triaxial ellipsoid in the papers (Bektaş, 2014; Bektaş, 2015; Ligas, 2012). Using other ways of parameterization of a triaxial ellipsoid may simplify the process of creating map projections and enable a more aesthetic form of the functions. For example Nyrtsov (2014) used ellipsoidal coordinates to create conformal projection of a triaxial ellipsoid and Bugayevsky (1991) used isometric coordinates for the same purpose. The author of this paper propose to use reduced coordinates in equidistant map projections. Presentation of the equation of a triaxial ellipsoid in reduced coordinates provides the opportunity to derive simple equations for the calculation of lengths of meridians and parallels and to construct map projections with use of the normal elliptic integrals of the second kind and Jacobian elliptic functions. In practice, the task that is necessary in 
the application of this type of mapping is to carry out the transformation between coordinate systems of a triaxial ellipsoid. Well-known and relatively simple equations allow to make such calculations. The transformation between planetographic, planetocentric and reduced coordinate systems is carried out according to the following scheme:

$$
\begin{aligned}
& (u, v) \Leftrightarrow(X, Y, Z) \Leftrightarrow(B, L) \\
& \text { \ } \\
& (\varphi, \lambda)
\end{aligned}
$$

where: $X, Y, Z-3 \mathrm{D}$ Cartesian coordinates, $u-$ a reduced latitude, $v-$ a reduced longitude, $\varphi-$ a planetocentric latitude, $\lambda-$ a planetocentric longitude, $B-$ a planetographic latitude, L.- a planetographic longitude. According to the scheme in order to transform the coordinates from one system to another, the latitudes and longitudes are transformed to 3D Cartesian coordinates $X, Y, Z$ first and then to latitudes and longitudes in the other system. So it is necessary to know the equations for direct and inverse transformation between planetographic, planetocentric, reduced coordinate systems and the 3D Cartesian coordinate system.

The main aim of the paper is to present the construction of equidistant map projections of a triaxial ellipsoid with use of reduced coordinates. The equations for the lengths of meridians and parallels are expressed by means of the normal elliptic integrals of the second kind and Jacobian elliptic functions.

The article starts with a presentation of the reduced coordinate system of a triaxial ellipsoid. This section contains the equations of transformations to 3D Cartesian coordinates and properties of a graticule. The next section demonstrates the derivation of equations for lengths of meridians and parallels with use of the normal elliptic integrals of the second kind and Jacobian elliptic functions. The subsequent section describes equidistant along meridians map projections of a triaxial ellipsoid in reduced coordinates and presents the images of graticules of a triaxial ellipsoid. The developed projections may be applied to create maps of extraterrestrial objects.

\section{Reduced coordinates}

The reduced coordinates on an oblated ellipsoid are commonly known in geodesy. This section describes how to generalize them to a triaxial ellipsoid. The term "reduced" latitude is used in case of an oblated ellipsoid and planetocentric, planetographic and reduced latitudes are distinguished as different coordinates. However, as far as an oblated ellipsoid is concerned, the longitude is the same for these three systems. In case of a triaxial ellipsoid there are differences both in latitudes and longitudes between those three coordinate systems. So in this paper, the authors use the term "reduced" for latitude and longitude in case of a triaxial 
ellipsoid and consider them a generalization of reduced coordinates of an oblated ellipsoid to a triaxial ellipsoid. In literature, reduced coordinates on a triaxial ellipsoid are sometimes referred to as parametric coordinates (Bektaş, 2015) and equations of a triaxial ellipsoid in reduced coordinates are known as a Gaussian parametric form (Bektaş,2015).

The relationship between the 3D Cartesian coordinates and the reduced coordinates of a triaxial ellipsoid is represented by equations (Bektaş, 2015):

$$
\begin{aligned}
& X=a \cos u \cos v \\
& Y=b \cos u \sin v \\
& Z=c \sin u
\end{aligned}
$$

where:

$a, b, c$ are the semi-axes of a triaxial ellipsoid,

$u$ is the reduced latitude,

$v$ is the reduced longitude.

The dependence that enables to determine the reduced coordinates on the basis of the 3D Cartesian coordinates has the following form:

$$
\begin{aligned}
& u=\arcsin \frac{Z}{c}, \\
& v=\arctan \frac{a Y}{b X} .
\end{aligned}
$$

The view of the reduced coordinate graticule on a triaxial ellipsoid is shown in fig. 1. Parallels are drawn with an interval $\Delta u=10^{\circ}$, meridians also with $\Delta v=10^{\circ}$. Meridians in a reduced coordinates system are ellipses with the length of the polar semi-axis equal to $c$ and the length of the equatorial semi-axis varying from $a$ to $b$ depending on the parameter $v$. Parallels are also ellipses with the length of the first semi-axis equals to $a$ consu and the second equals to $b$ consu. Meridians $v=$ const and parallels $u=$ const are not orthogonal (Bektaş, 2015). They are shown in Figure 1. 


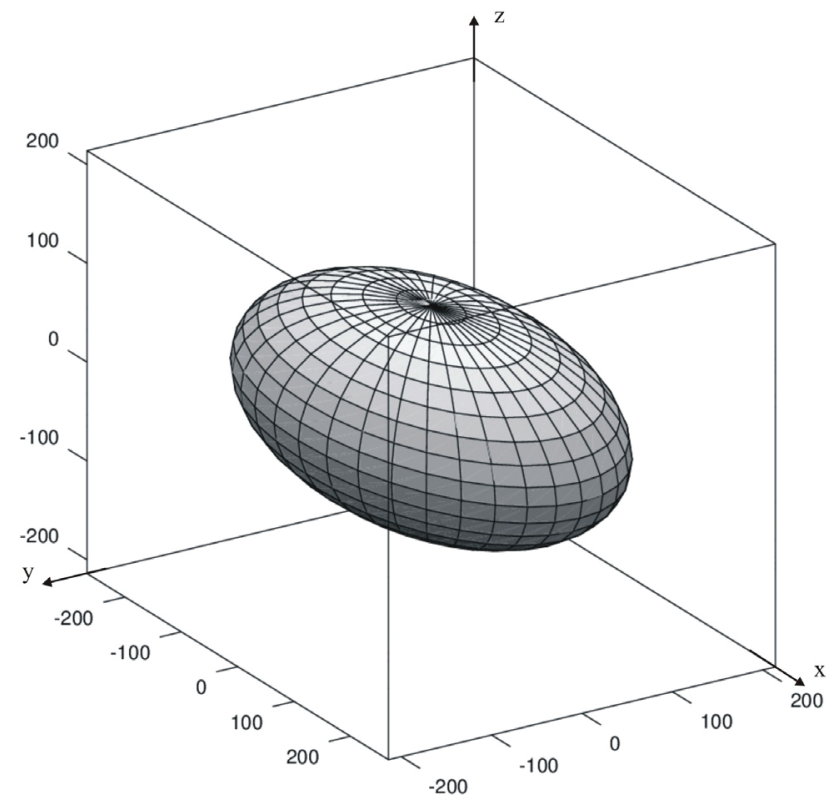

Fig. 1. View of the reduced coordinate graticule on a triaxial ellipsoid in perspective. Parallels are drawn with interval $\Delta u=10^{\circ}$, meridians also with $\Delta v=10^{\circ}$.

\section{The lengths of meridians and parallels of a triaxial ellipsoid in reduced coordinates}

In order to construct an equidistant along meridians and parallels map projections the equations for the lengths of meridians and parallels are required. On a triaxial ellipsoid the parallels $u=$ const are ellipses. On a parallel the coordinate $v$ changes. The length of arc of parallel is calculated from the central meridian to any other meridian. On a triaxial ellipsoid meridians $v=$ const are ellipses too. On a meridian the coordinate $u$ changes. The length of the arc of a meridian is calculated from the equator to any other parallel. Equations are derived basing on the equation of a triaxial ellipsoid in the form (1) where reduced coordinates are used.

\subsection{Derivation of equations for the lengths of meridians between the equator and a parallel}

The length of the arc of a meridian $s_{m}$ between the equator $u=0$ and the parallel $u=u_{i}$ on a triaxial ellipsoid described by equation (1) is expressed by the equation: 


$$
s_{m}=\int_{0}^{u_{i}} \sqrt{\left(\frac{d X}{d u}\right)^{2}+\left(\frac{d Y}{d u}\right)^{2}+\left(\frac{d Z}{d u}\right)^{2}} d u
$$

where the partial derivatives are in the form:

$\frac{d X}{d u}=-a \sin u \cos v$

$\frac{d Y}{d u}=-b \sin u \sin v$

$\frac{d Z}{d u}=c \cos u$

and they are obtained by differentiation of (1). In that case the reduced latitude $u$ is variable and the reduced longitude $v$ is constant. After substituting partial derivatives to (3) some modification is introduced:

$$
\begin{aligned}
S_{m} & =\int_{0}^{u_{i}} \sqrt{a^{2} \sin ^{2} u \cos ^{2} v+b^{2} \sin ^{2} u \sin ^{2} v+c^{2} \cos ^{2} u} d u= \\
& =\int_{0}^{u_{i}} \sqrt{a^{2} \sin ^{2} u \cos ^{2} v+b^{2} \sin ^{2} u \sin ^{2} v-c^{2} \sin ^{2} u+c^{2}} d u= \\
& =\int_{0}^{u_{i}} \sqrt{\sin ^{2} u\left(a^{2} \cos ^{2} v+b^{2} \sin ^{2} v-c^{2}\right)+c^{2}} d u= \\
& =c \int_{0}^{u_{i}} \sqrt{1+\sin ^{2} u\left(\frac{a^{2} \cos ^{2} v+b^{2} \sin ^{2} v}{c^{2}}-1\right)} d u
\end{aligned}
$$

Finally the equation for the length of a meridian is obtained, in the form of:

$$
S_{m}=c \int_{0}^{u_{i}} \sqrt{1+n_{m}{ }^{2} \sin ^{2} u} d u=c I_{1 m},
$$

where:

$$
n_{m}{ }^{2}=\frac{a^{2} \cos ^{2} v+b^{2} \sin ^{2} v}{c^{2}}-1 .
$$

If $a>b>c$ then $n_{m}{ }^{2}>0$ and according to (Byrd and Friedmann, 1954) the integral $I_{1 m}$ can be presented in the form of: 
$I_{1 m}=k_{m}^{\prime} I_{2 m}$

where:

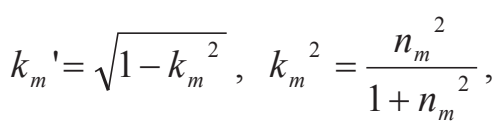

and the integral $I_{2 m}$ has the following form (Byrd and Friedmann, 1954):

$$
I_{2 m}=\frac{1}{k_{m}{ }^{2}}\left[E\left(\psi_{m}, k_{m}\right)-k_{m}{ }^{2} \operatorname{sn} \vartheta_{m} \operatorname{cd} \vartheta_{m}\right]
$$

where:

$E\left(\psi_{m}, k_{m}\right)$ is the normal elliptic integral of the second kind,

$\psi_{m}=\arcsin \left(\sqrt{\frac{\left(1+n_{m}{ }^{2}\right) \sin ^{2} u}{1+n_{m}{ }^{2} \sin ^{2} u}}\right), \operatorname{sn} \vartheta_{m}=\sqrt{\frac{\left(1+n_{m}{ }^{2}\right) \sin ^{2} u}{1+n_{m}{ }^{2} \sin ^{2} u}}, \operatorname{cd} \vartheta_{m}=\sqrt{\frac{1-\operatorname{sn}^{2} \vartheta_{m}}{1-k_{m}{ }^{2} \operatorname{sn}^{2} \vartheta_{m}}}$,

$\operatorname{sn} \vartheta_{m}$ and $\operatorname{cd} \vartheta_{m}$ are Jacobian elliptic functions.

Finally, the equation for the length of a meridian arc is as follows:

$$
s_{m}=c \frac{1}{k_{m}{ }^{\prime}}\left[E\left(\psi_{m}, k_{m}\right)-k_{m}{ }^{2} \operatorname{sn} \vartheta_{m} \operatorname{cd} \vartheta_{m}\right]
$$

where:

$$
\begin{aligned}
& k_{m}{ }^{\prime}=\sqrt{1-k_{m}{ }^{2}}, k_{m}{ }^{2}=\frac{n_{m}{ }^{2}}{1+n_{m}{ }^{2}}, n_{m}{ }^{2}=\frac{a^{2} \cos ^{2} v+b^{2} \sin ^{2} v}{c^{2}}-1, \operatorname{sn} \vartheta_{m}=\sqrt{\frac{\left(1+n_{m}{ }^{2}\right) \sin ^{2} u}{1+n_{m}{ }^{2} \sin ^{2} u},} \\
& \operatorname{cd} \vartheta_{m}=\sqrt{\frac{1-\operatorname{sn}^{2} \vartheta_{m}}{1-k_{m}{ }^{2} \operatorname{sn}^{2} \vartheta_{m}}}, \psi_{m}=\arcsin \left(\sqrt{\frac{\left(1+n_{m}{ }^{2}\right) \sin ^{2} u}{1+n_{m}{ }^{2} \sin ^{2} u}}\right) .
\end{aligned}
$$

\subsection{Derivation of the equations for the lengths of parallels between the central meridian and any other meridian}

The equation for the length of the parallel arc $s_{p}$ between central meridian $v=0$ and meridian $v=v_{i}$ on a triaxial ellipsoid described by equation (1) is as follows: 


$$
s_{p}=\int_{0}^{v_{i}} \sqrt{\left(\frac{d X}{d v}\right)^{2}+\left(\frac{d Y}{d v}\right)^{2}+\left(\frac{d Z}{d v}\right)^{2}} d v
$$

where the partial derivatives are in the form:

$$
\begin{aligned}
& \frac{d X}{d v}=-a \cos u \sin v \\
& \frac{d Y}{d v}=b \cos u \cos v \\
& \frac{d Z}{d v}=0
\end{aligned}
$$

and they are obtained by differentiation of (1). In that case the reduced longitude $v$ is variable and the reduced latitude $u$ is constant. After substituting partial derivatives to (5) some modification is introduced:

$$
\begin{aligned}
& s_{p}=\int_{0}^{v_{i}} \sqrt{a^{2} \cos ^{2} u \sin ^{2} v+b^{2} \cos ^{2} u \cos ^{2} v} d v= \\
& =b \cos u \int_{0}^{v_{i}} \sqrt{1-\sin ^{2} v+\frac{a^{2}}{b^{2}} \sin ^{2} v} d v= \\
& =b \cos u \int_{0}^{v_{i}} \sqrt{1+\sin ^{2} v\left(\frac{a^{2}}{b^{2}}-1\right)} d v
\end{aligned}
$$

Finally the equation for length of a parallel is obtained, in the form of:

$$
s_{p}=b \cos u \int_{0}^{v_{i}} \sqrt{1+n_{p}{ }^{2} \sin ^{2} v} d v=b I_{1 p}
$$

where:

$$
n_{p}^{2}=\frac{a^{2}}{b^{2}}-1 \text {. }
$$

If $a>b$ then $n_{p}{ }^{2}>0$ and the integral $I_{1 p}$, similarly as for a meridian can be presented in the form of (Byrd and Friedmann, 1954):

$$
I_{1 p}=k_{p}^{\prime} I_{2 p}
$$

where: 
$k_{p}^{\prime}=\sqrt{1-k_{p}^{2}}, k_{p}^{2}=\frac{n_{p}^{2}}{1+n_{p}^{2}}$,

and integral $I_{2 p}$ has the following form (Byrd and Friedmann, 1954):

$I_{2 p}=\frac{1}{k_{p}{ }^{2}}\left[E\left(\psi_{p}, k_{p}\right)-k_{p}^{2} \operatorname{sn} \vartheta_{p} \operatorname{cd} \vartheta_{p}\right]$

where:

$E\left(\psi_{p}, k_{p}\right)$ is the normal elliptic integral of the second kind,

$\psi_{p}=\arcsin \left(\sqrt{\frac{\left(1+n_{p}^{2}\right) \sin ^{2} v}{1+n_{p}^{2} \sin ^{2} v}}\right), \operatorname{sn} \vartheta_{p}=\sqrt{\frac{\left(1+n_{p}^{2}\right) \sin ^{2} v}{1+n_{p}^{2} \sin ^{2} v}}, \operatorname{cd} \vartheta_{p}=\sqrt{\frac{1-\operatorname{sn}^{2} \vartheta_{p}}{1-k_{p}^{2} \operatorname{sn}^{2} \vartheta_{p}}}$

$\operatorname{sn} \vartheta_{p}$ and $\operatorname{cd} \vartheta_{p}$ are Jacobian elliptic functions.

Finally, the length of the parallel's arc can be expressed using the equation:

$$
s_{p}=b \cos u \frac{1}{k_{p}{ }^{l}}\left[E\left(\psi_{p}, k_{p}\right)-k_{p}^{2} \operatorname{sn} \vartheta_{p} \operatorname{cd} \vartheta_{p}\right]
$$

where:

$E\left(\psi_{p}, k_{p}\right)$ is the normal elliptic integral of the second kind,

$$
\begin{aligned}
& k_{p}^{\prime}=\sqrt{1-k_{p}^{2}}, k_{p}^{2}=\frac{n_{p}{ }^{2}}{1+n_{p}{ }^{2}}, n_{p}{ }^{2}=\frac{a^{2}}{b^{2}}-1, \operatorname{sn} \vartheta_{p}=\sqrt{\frac{\left(1+n_{p}{ }^{2}\right) \sin ^{2} v}{1+n_{p}{ }^{2} \sin ^{2} v}} \\
& \operatorname{cd} \vartheta_{p}=\sqrt{\frac{1-\operatorname{sn}^{2} \vartheta_{p}}{1-k_{p}{ }^{2} \operatorname{sn}^{2} \vartheta_{p}}}, \psi_{p}=\arcsin \left(\sqrt{\frac{\left(1+n_{p}{ }^{2}\right) \sin ^{2} v}{1+n_{p}{ }^{2} \sin ^{2} v}}\right) .
\end{aligned}
$$

\subsection{The results of calculations of the lengths of meridians}

Due to the fact that the meridians and parallels in a reduced coordinate system are ellipses, similar algorithms may be used to calculate the lengths of their arcs. Equations (4) and (6) differ slightly, so the author decided to test only the algorithm 
for the calculation of lengths of meridians. Moreover, this algorithm may be used to calculate the lengths of meridians in other coordinate systems, i.e. planetographic and planetocentric, because they are also ellipses but located on different planes. In that case it is required to transform the coordinates from planetographic or planetocentric system to the reduced coordinate system and then the lengths of meridians may be calculated. Thus, it is possible to control the results obtained with use of equation (4) by using the equation for the length of a meridian on a triaxial ellipsoid expressed by planetocentric or planetographic coordinates.

Using the derived equation (4) calculations were performed for $1 / 8$ part of a triaxial ellipsoid, where $u \in\left\langle 0, \frac{\pi}{2}\right\rangle, v \in\left\langle 0, \frac{\pi}{2}\right\rangle$. The other parts of an ellipsoid are symmetrical. For the purposes of calculations the following lengths of the semi-axis were assumed: $a=267.5 \mathrm{~m}, b=147 \mathrm{~m}, c=104.5 \mathrm{~m}$ for a triaxial ellipsoid as a reference surface for asteroid 25143 Itokawa (Nyrtsov at al., 2014). First of all, the reduced and planetocentric coordinates were calculated for 16 points located on a triaxial ellipsoid. Points were located on 4 meridians $v=0^{\circ}, 30^{\circ}, 60^{\circ}$ and $90^{\circ}$ and four parallels $u=0^{\circ}, 30^{\circ}, 60^{\circ}$ and $90^{\circ}$ and the coordinates were given in reduced coordinate system. Then planetocentric coordinates were calculated for these points. This means that first the 3D Cartesian coordinates were calculated using equation (1) and then the planetocentric coordinates were calculated using equations (7) obtained on the basis of equation of a triaxial ellipsoid given in (Nyrtsov at al., 2007). The results are presented in Table 1 in columns $1-5$.

$$
\begin{aligned}
& \sin \varphi=\frac{Z}{\rho}, \\
& \tan \lambda=\frac{Y}{X} .
\end{aligned}
$$

where:

$\rho=\sqrt{X^{2}+Y^{2}+Z^{2}}$

$X, Y, Z-3 \mathrm{D}$ Cartesian coordinates, $\varphi$ is a planetocentric latitude, $\lambda-$ a planetocentric longitude.

Then the lengths of the arcs of meridians between points from table 1 were calculated. It means that the lengths of 12 arcs of meridians $v=0^{\circ}, 30^{\circ}, 60^{\circ}$ and $90^{\circ}$ ( 3 arcs for every meridian between the equator $u=0^{\circ}$ and parallels $u=30^{\circ}, 60^{\circ}$ and $90^{\circ}$ ) were calculated. For this calculation equations (4) were used. The results are presented in the $7^{\text {th }}$ column of Table 1 . The lengths of these arcs of meridians were verified by their calculation using numerical integration with alternative following equations (http://geocnt.geonet.ru/en/3_axial): 


$$
S_{m}=c \int_{0}^{\varphi} \frac{\sqrt{1-\left(2-e_{d}{ }^{2}\right) e_{d}{ }^{2} \cos ^{2} \varphi}}{\left(1-e_{d}{ }^{2} \cos ^{2} \varphi\right)^{\frac{3}{2}}} d \varphi
$$

where:

$\varphi$ is a planetocentric latitude,

$\lambda$ is a planetocentric longitude,

$$
e_{1}^{2}=\left(a^{2}-b^{2}\right) / a^{2}, e_{d}^{2}=\left(d_{e l}^{2}-c^{2}\right) / d_{e l}^{2}, d_{e l}=\frac{b}{\sqrt{1-e_{1}^{2} \cos ^{2} \lambda}}
$$

The arcs of meridians were calculated basing on the planetocentric coordinates from column 4 and 5 of Table 1 and equation (8). The results are listed in the $8^{\text {th }}$ column of Table 1. So the lengths of meridians were determined in two coordinate systems (with different longitudes) but located on the same appropriate planes. The arcs of the same ellipses were calculated with the use of different equations.

The results are shown in Table 1. The length of arcs of meridians was calculated with use of equations 4 and 8 .

\begin{tabular}{|c|c|c|c|c|c|c|c|}
\hline points & reduced & coordinates & planetocentric & coordinates & line & $\begin{array}{l}\text { lengths of } \\
\text { meridians } \\
\text { in reduced } \\
\text { coordinates } \\
{[\mathrm{m}]}\end{array}$ & $\begin{array}{l}\text { lengths of } \\
\text { meridians in } \\
\text { planetocentric } \\
\text { coordinates } \\
{[\mathrm{m}]}\end{array}$ \\
\hline & $u$ & $v$ & $\varphi$ & $\lambda$ & & & \\
\hline 1 & 2 & 3 & 4 & 5 & 6 & 7 & 8 \\
\hline $\mathrm{P}_{1}$ & 0 & 0 & 0 & 0 & & & \\
\hline $\mathrm{P}_{2}$ & 30 & 0 & 12.71006 & 0 & $\mathrm{P}_{1} \mathrm{P}_{2}$ & 65.930 & 65.930 \\
\hline $\mathrm{P}_{3}$ & 60 & 0 & 34.08358 & 0 & $\mathrm{P}_{1} \mathrm{P}_{3}$ & 171.620 & 171.620 \\
\hline $\mathrm{P}_{4}$ & 90 & 0 & 90 & 0 & $\mathrm{P}_{1} \mathrm{P}_{4}$ & 306.369 & 306.369 \\
\hline $\mathrm{P}_{5}$ & 0 & 30 & 0 & 17.60282 & & & \\
\hline $\mathrm{P}_{6}$ & 30 & 30 & 13.94137 & 17.60282 & $\mathrm{P}_{5} \mathrm{P}_{6}$ & 63.860 & 63.860 \\
\hline $\mathrm{P}_{7}$ & 60 & 30 & 36.67595 & 17.60282 & $\mathrm{P}_{5} \mathrm{P}_{7}$ & 161.296 & 161.296 \\
\hline $\mathrm{P}_{8}$ & 90 & 30 & 90 & 17.60282 & $\mathrm{P}_{5} \mathrm{P}_{8}$ & 283.915 & 283.915 \\
\hline $\mathrm{P}_{9}$ & 0 & 60 & 0 & 43.58592 & & & \\
\hline $\mathrm{P}_{10}$ & 30 & 60 & 18.09440 & 43.58592 & $\mathrm{P}_{9} \mathrm{P}_{10}$ & 59.394 & 59.394 \\
\hline $\mathrm{P}_{11}$ & 60 & 60 & 44.42789 & 43.58592 & $\mathrm{P}_{9} \mathrm{P}_{11}$ & 137.720 & 137.720 \\
\hline $\mathrm{P}_{12}$ & 90 & 60 & 90 & 43.58592 & $\mathrm{P}_{9} \mathrm{P}_{12}$ & 231.482 & 231.482 \\
\hline $\mathrm{P}_{13}$ & 0 & 90 & 0 & 90 & & & \\
\hline $\mathrm{P}_{14}$ & 30 & 90 & 22.31468 & 90 & $\mathrm{P}_{13} \mathrm{P}_{14}$ & 56.953 & 56.953 \\
\hline $\mathrm{P}_{15}$ & 60 & 90 & 50.91795 & 90 & $\mathrm{P}_{13} \mathrm{P}_{15}$ & 123.651 & 123.651 \\
\hline $\mathrm{P}_{16}$ & 90 & 90 & 90 & 90 & $\mathrm{P}_{13} \mathrm{P}_{16}$ & 198.940 & 198.940 \\
\hline
\end{tabular}

Table 1. The results of calculations of coordinates and lengths of meridians for a triaxial ellipsoid 
There are significant differences between the calculated coordinates in the two coordinate systems: planetocentric and reduced. The meridians and parallels of these systems do not coincide with each other because they reflect different coordinate systems. Meridians in both coordinate systems are ellipses located on different planes but it was found that such longitudes where meridians were located on the same ellipses, which enabled us to perform calculations. The same results were obtained for the calculation (with millimeter accuracy) of the lengths of the arcs of meridians using two equations (4) and (8). Thus, the derived equations (4) can be considered correct.

\section{Equidistant map projections in reduced coordinates}

In this section equidistant along meridians and parallels map projections of a triaxial ellipsoid are described. First of all, the cylindrical projection is presented. This type of projection may be used to present entire extraterrestrial objects. In such projection meridians are projected as straight parallel lines, while parallels are projected as curves. If the assumed equatorial eccentricity is equal to 0 then the commonly known cylindrical projection of an oblated ellipsoid is obtained. Then pseudocylindrical projection is described. This type of projection may also be used to present entire extraterrestrial objects. It is similar to pseudocylindrical projection of a oblated ellipsoid. In this projection, the parallels (lines of constant reduced latitudes) are projected as straight parallel lines. If the assumed equatorial eccentricity is equal to 0 then the result is a pseudocylindrical projection of an oblated ellipsoid. In the end an azimuthal projection of a triaxial ellipsoid is presented. This type of projection may be used to present the polar regions of extraterrestrial objects. In this projection, the meridians are projected as straight radiant lines intersecting at a single point, while parallels are projected as curves (not circles as in the azimuthal projections of an oblated ellipsoid). If the assumed equatorial eccentricity is equal to 0 then the commonly known azimuthal projection of an oblated ellipsoid is obtained. All the discussed projections are based on the previously derived equations (4) and (6) for lengths of meridians and parallels.

\subsection{Cylindrical map projection equidistant along meridians}

Cylindrical map projections of a triaxial ellipsoid are different from cylindrical map projections of an oblated ellipsoid or a sphere. Meridians (lines of constant reduced longitudes) are projected as straight parallel lines, while parallels are projected as curves, except the equator, which is projected as a straight line and is perpendicular to the images of meridians. The spacing of meridians is preserved along the image of the equator. The general equation for a cylindrical projection of a triaxial ellipsoid is as follows: 


$$
x=f_{1}(u), y=f_{2}(v) .
$$

The equidistant along meridians cylindrical map projections is constructed basing on the assumption that the $x$ coordinate is equal to the length of meridian which is provided by equation (4) and that the $y$ coordinate is equal to the length of the equator which is the result of equation (6) with $u=0$. As a result of the second assumption, the equator is projected with preserved spacing of meridians.

Using equations (4) and (6), respectively, for the lengths of meridians and parallels, the cylindrical equidistant map projection equations can be written as:

$$
x=c \frac{1}{k_{m}{ }^{\prime}}\left[E\left(\psi_{m}, k_{m}\right)-k_{m}{ }^{2} \operatorname{sn} \vartheta_{m} \operatorname{cd} \vartheta_{m}\right]
$$

where:

$k_{m}{ }^{\prime}=\sqrt{1-k_{m}{ }^{2}}, \quad k_{m}{ }^{2}=\frac{n_{m}{ }^{2}}{1+n_{m}{ }^{2}}, \quad n_{m}{ }^{2}=\frac{a^{2} \cos ^{2} v+b^{2} \sin ^{2} v}{c^{2}}-1$,

$\operatorname{sn} \vartheta_{m}=\sqrt{\frac{\left(1+n_{m}{ }^{2}\right) \sin ^{2} u}{1+n_{m}{ }^{2} \sin ^{2} u}}$,

$\operatorname{cd} \vartheta_{m}=\sqrt{\frac{1-\operatorname{sn}^{2} \vartheta_{m}}{1-k_{m}{ }^{2} \operatorname{sn}^{2} \vartheta_{m}}}, \psi_{m}=\arcsin \left(\sqrt{\frac{\left(1+n_{m}{ }^{2}\right) \sin ^{2} u}{1+n_{m}{ }^{2} \sin ^{2} u}}\right)$.

and

$$
y=b \frac{1}{k_{p}{ }^{\prime}}\left[E\left(\psi_{p}, k_{p}\right)-k_{p}{ }^{2} \operatorname{sn} \vartheta_{p} \operatorname{cd} \vartheta_{p}\right]
$$

where:

$k_{p}{ }^{\prime}=\sqrt{1-k_{p}{ }^{2}}, k_{p}{ }^{2}=\frac{n_{p}{ }^{2}}{1+n_{p}{ }^{2}}, n_{p}{ }^{2}=\frac{a^{2}}{b^{2}}-1, \operatorname{sn} \vartheta_{p}=\sqrt{\frac{\left(1+n_{p}{ }^{2}\right) \sin ^{2} v}{1+n_{p}{ }^{2} \sin ^{2} v}}$,

$\operatorname{cd} \vartheta_{p}=\sqrt{\frac{1-\operatorname{sn}^{2} \vartheta_{p}}{1-k_{p}{ }^{2} \operatorname{sn}^{2} \vartheta_{p}}}, \psi_{p}=\arcsin \left(\sqrt{\frac{\left(1+n_{p}{ }^{2}\right) \sin ^{2} v}{1+n_{p}{ }^{2} \sin ^{2} v}}\right)$.

The graticules of the reduced coordinates of the entire and of a fragment of a triaxial ellipsoid in the developed map projection are shown in Figure 2. 

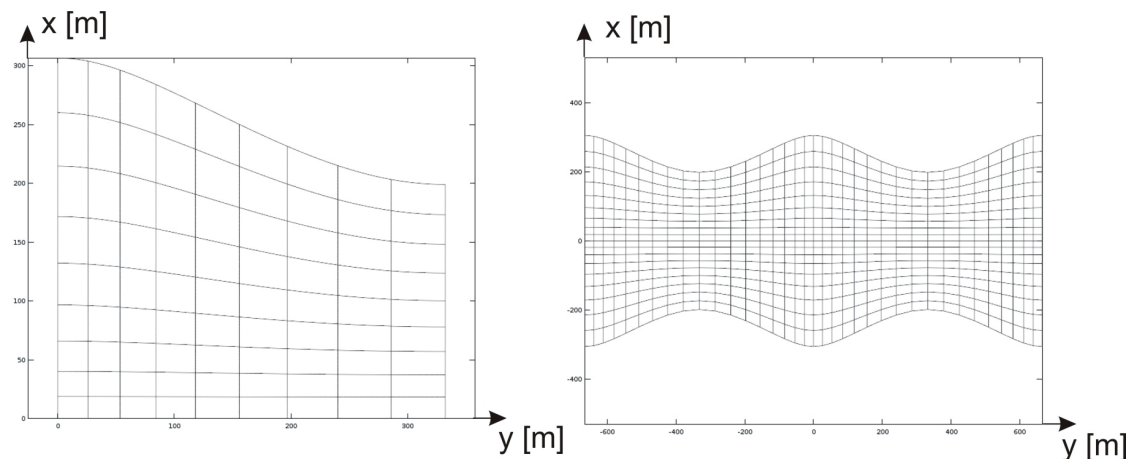

Fig. 2. The graticule of reduced coordinates of the entire and 1/8 triaxial ellipsoid in the developed map projection

Images of meridians and parallels of reduced coordinates in a cylindrical equidistant along meridians projection are not orthogonal to each other. Images of meridians form straight lines parallel to the $\mathrm{x}$-axis of the Cartesian coordinate system. They have different lengths. The distances between the images of meridians are variables and they correspond to the distances between the meridians along the equator on an ellipsoid. The lengths of meridians are variables, so the graticule limited by curves to the south and north. The images of parallels are curves except the equator, which is projected as straight line. Figure 2 presents the graticule of reduced coordinates on a triaxial ellipsoid. However, such graticule is not used in practice. Planetocentric or planetographic graticules are typically used on maps of extraterrestrial objects. So such graticules were drawn. In the developed map projection, the graticules of reduced, planetographic and planetocentric coordinates are shown in Figure 3. Meridians in three coordinate systems are projected as parallel straight lines but they don't overlap except meridians with $v, L$ and $\lambda$ equal to $0^{\circ}, 90^{\circ},-90^{\circ},-180^{\circ}$. The lengths are preserved in meridional direction. Parallels of three coordinates systems are projected as differently shaped curves, except the equator which is projected as a straight line and the poles which are projected as overlapping curves.
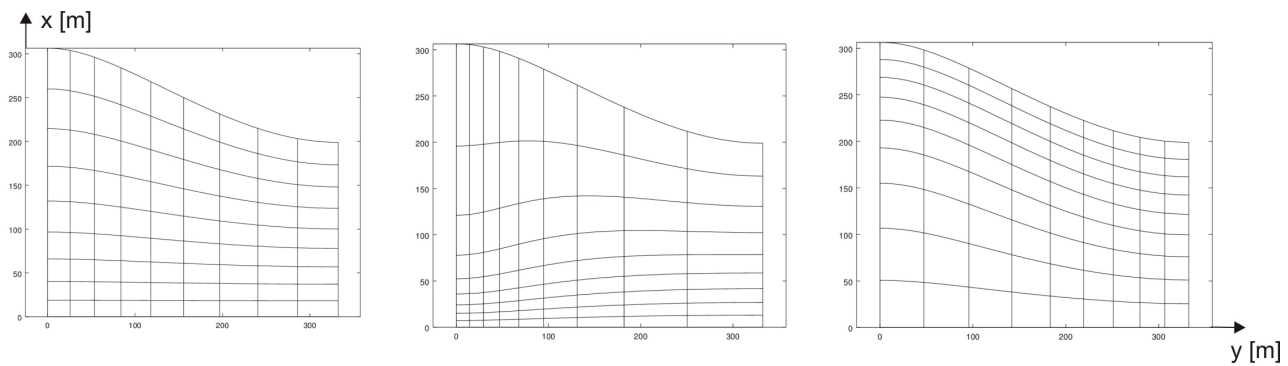

Fig. 3. Graticules in a cylindrical map projection, left - reduced coordinates, middle - planetographic coordinates, right - planetocentric coordinates 


\subsection{Pseudocylindrical map projection equidistant along parallels (lines of constant reduced latitudes)}

Another interesting map projection based on equations (4) and (6) for the lengths of meridians and parallels derived in this paper, is the pseudocylindrical projection. In this type of projection of a triaxial ellipsoid the parallels are projected on a plane as straight lines and their lengths are preserved, while the meridians are projected as curves. General equations for coordinates $x$ and $y$ have the following form:

$$
x=f_{1}(u), y=f_{2}(u, v) .
$$

To construct equidistant along parallels pseudocylindrical map projection it was assumed that the $x$ coordinate is equal to length of central meridian which is provided by equation (4) with $v=0$ and that the $y$ coordinate is equal to the length of parallel which is provided by equation (6). As a result of the first assumption, the central meridian is projected with preserved spacing of parallels.

Using equations (4) and (6), respectively, to calculate the lengths of meridians and parallels pseudocylindrical equidistant along parallels map projection can be written as:

$$
x=c \frac{1}{k_{m}{ }^{\prime}}\left[E\left(\psi_{m}, k_{m}\right)-k_{m}{ }^{2} \operatorname{sn} \vartheta_{m} \operatorname{cd} \vartheta_{m}\right]
$$

where:

$$
\begin{aligned}
& k_{m}{ }^{\prime}=\sqrt{1-k_{m}{ }^{2}}, k_{m}{ }^{2}=\frac{n_{m}{ }^{2}}{1+n_{m}{ }^{2}}, n_{m}{ }^{2}=\frac{a^{2}}{c^{2}}-1, \operatorname{sn} \vartheta_{m}=\sqrt{\frac{\left(1+n_{m}{ }^{2}\right) \sin ^{2} u}{1+n_{m}{ }^{2} \sin ^{2} u}}, \\
& \operatorname{cd} \vartheta_{m}=\sqrt{\frac{1-\operatorname{sn}^{2} \vartheta_{m}}{1-k_{m}{ }^{2} \operatorname{sn}^{2} \vartheta_{m}}}, \psi_{m}=\arcsin \left(\sqrt{\frac{\left(1+n_{m}{ }^{2}\right) \sin ^{2} u}{1+n_{m}{ }^{2} \sin ^{2} u}}\right),
\end{aligned}
$$

and

$$
y=b \cos u \frac{1}{k_{p}{ }^{l}}\left[E\left(\psi_{p}, k_{p}\right)-k_{p}{ }^{2} \operatorname{sn} \vartheta_{p} \operatorname{cd} \vartheta_{p}\right] \text {, }
$$

where:

$$
\begin{aligned}
& k_{p}{ }^{\prime}=\sqrt{1-k_{p}{ }^{2}}, k_{p}{ }^{2}=\frac{n_{p}{ }^{2}}{1+n_{p}{ }^{2}}, n_{p}{ }^{2}=\frac{a^{2}}{b^{2}}-1, \operatorname{sn} \vartheta_{p}=\sqrt{\frac{\left(1+n_{p}{ }^{2}\right) \sin ^{2} v}{1+n_{p}{ }^{2} \sin ^{2} v}}, \\
& \operatorname{cd} \vartheta_{p}=\sqrt{\frac{1-\operatorname{sn}^{2} \vartheta_{p}}{1-k_{p}{ }^{2} \operatorname{sn}^{2} \vartheta_{p}}} \psi_{p}=\arcsin \left(\sqrt{\frac{\left(1+n_{p}{ }^{2}\right) \sin ^{2} v}{1+n_{p}{ }^{2} \sin ^{2} v}}\right) .
\end{aligned}
$$

The graticule in pseudocylindrical projection is shown in Figure 4. 


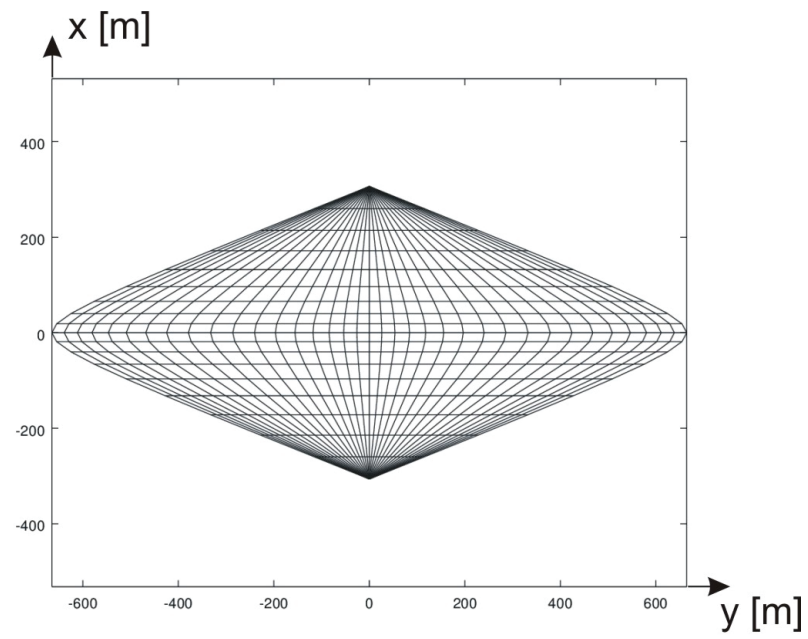

Fig. 4. The graticule in pseudocylindrical projection of the entire triaxial ellipsoid

This projection has an interesting property: the parallels $u=$ const are projected on straight lines parallel to the $y$ axis. Their lengths are preserved, as well as the distances between images of parallels along the central meridian. On the other hand, meridians are projected as curves. Figure 5 shows the graticules of three coordinate systems (reduced, planetographic, planetocentric) in a pseudocylindrical projection. Meridians in three coordinate systems are projected as curves with similar shapes but they don't overlap except meridians with $v, L$ and $\lambda$ equal to $0^{\circ}, 90^{\circ},-90^{\circ},-180^{\circ}$. Parallels of planetocentric and planetographic coordinate systems are projected as curves with different shapes. The images of parallels of reduced coordinate system are projected as straight parallel lines. The equator in these three coordinate systems is projected as a straight line and the poles as points.
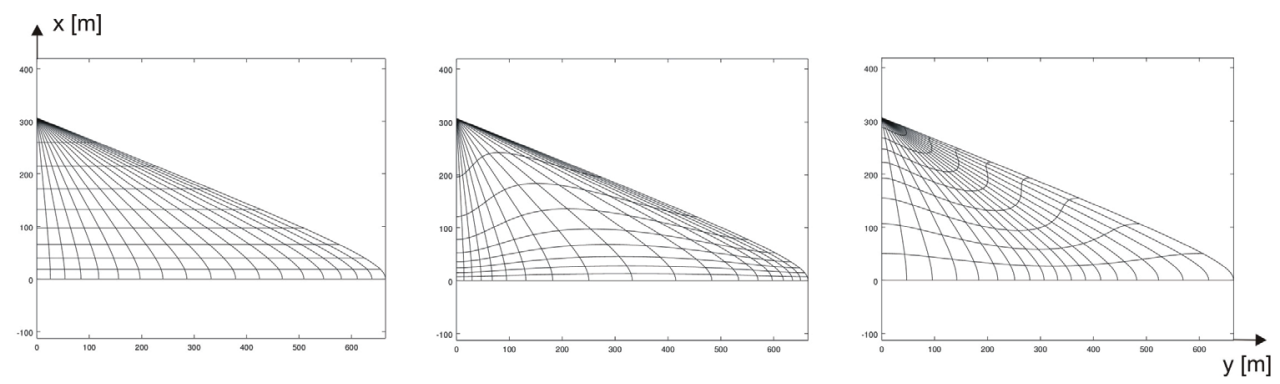

Fig. 5. Graticules of three coordinate systems in pseudocylindrical projection, left - reduced coordinates, middle - planetographic coordinates, right - planetocentric coordinates 


\subsection{Azimuthal map projection equidistant along meridians}

Another map projection, which can be constructed on the basis of the derived equation (4) for the lengths of meridians, is an equidistant along meridians azimuthal projection. In the azimuthal projection of a triaxial ellipsoid the meridians are projected as straight lines intersecting at a single point, while parallels are projected as curves. It has the following general form:

$$
x=\rho(u, v) \cos (v), y=\rho(u, v) \sin (v) .
$$

To construct equidistant along meridians azimuthal map projections it was assumed that the function $\rho$ is equal to length of meridians calculated from pole to parallel. In that case the length of the arc of a meridian given by equation (4) has to be

subtracted from the length of entire meridian which is equal to $\frac{c E\left(\frac{\pi}{2}, k_{m}\right)}{k_{m}{ }^{\prime}}$ according to (4) substituting $u=\frac{\pi}{2}$. Using equations (4) for lengths of meridians cylindrical equidistant map projection equations can be written as:

$$
x=\rho \cos (v), y=\rho \sin (v)
$$

where:

$$
\begin{aligned}
& \rho=c \frac{1}{k_{m}{ }^{\prime}}\left[E\left(\frac{\pi}{2}, k_{m}\right)-E\left(\psi_{m}, k_{m}\right)+k_{m}{ }^{2} \operatorname{sn} \vartheta_{m} \operatorname{cd} \vartheta_{m}\right], \\
& k_{m}{ }^{\prime}=\sqrt{1-{k_{m}}^{2}}, \quad{k_{m}}^{2}=\frac{n_{m}{ }^{2}}{1+n_{m}{ }^{2}}, \quad n_{m}{ }^{2}=\frac{a^{2} \cos ^{2} v+b^{2} \sin ^{2} v}{c^{2}}-1, \\
& \operatorname{sn} \vartheta_{m}=\sqrt{\frac{\left(1+n_{m}{ }^{2}\right) \sin ^{2} u}{1+n_{m}{ }^{2} \sin ^{2} u}}, \\
& \operatorname{cd} \vartheta_{m}=\sqrt{\frac{1-\operatorname{sn}^{2} \vartheta_{m}}{1-k_{m}{ }^{2} \operatorname{sn}^{2} \vartheta_{m}}}, \psi_{m}=\arcsin \left(\sqrt{\frac{\left(1+n_{m}{ }^{2}\right) \sin ^{2} u}{1+n_{m}{ }^{2} \sin ^{2} u}}\right) .
\end{aligned}
$$

The image of the reduced coordinate graticule will have the form shown in Figure 6. 


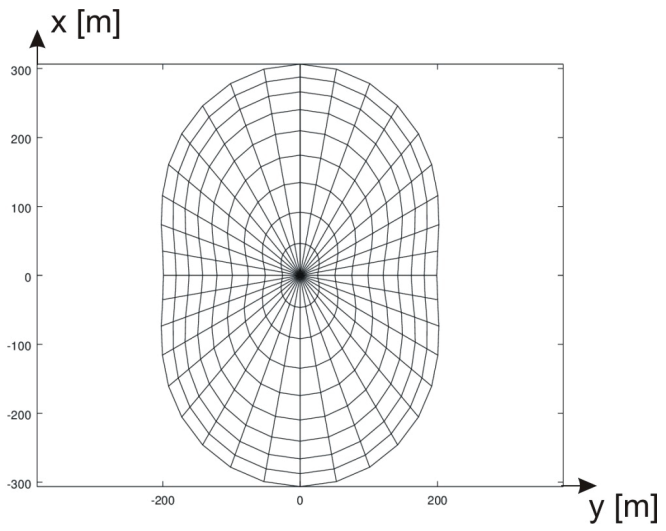

Fig. 6. Reduced coordinate graticule in azimuthal equidistant projection

The graticule consists of images of meridians which are straight lines and images of parallels which are curves. Images of parallels are not elliptical. In order to obtain images of parallels as ellipses, the projection functions must be modified by substituting $\lambda$ in the place of $v$ :

$$
x=\rho \cos (\lambda), y=\rho \sin (\lambda),
$$

where:

$\tan \lambda=\frac{b}{a} \tan v$.

Then the graticules will take such form as shown in Figure 7. So in that case of projection meridians are projected as straight lines and parallels as ellipses. In these three coordinate systems (planetocentric, planetographic and reduced) the meridians and parallels do not overlap except meridians with $v, L$ and $\lambda$ equal to $0^{\circ}, 90^{\circ},-90^{\circ}$, $-180^{\circ}$ and the equator.
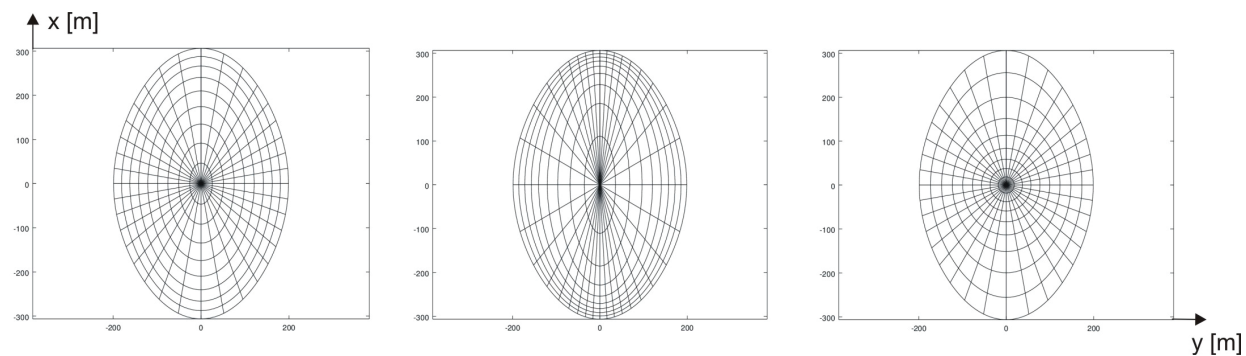

Fig. 7. Graticules in an equidistant along meridians azimuthal map projection of the half of a triaxial ellipsoid, left - reduced coordinates, middle - planetographic coordinates, right - planetocentric coordinates 


\section{Conclusions}

A triaxial ellipsoid is used as a reference surface for celestial objects with irregular shapes. Commonly used map projections of a triaxial ellipsoid are usually expressed as functions of planetographic or planetocentric coordinates. In this paper the authors used reduced coordinates to describe a triaxial ellipsoid and map projections. It allowed us to express map projections with use of the normal elliptic integral of the second kind and elliptic functions. The main advantage of this method is the fact that the calculations of $x$ and $y$ coordinates are practically based on a single algorithm that is required to solve the elliptic integral of the second kind. Equations for lengths of meridians and parallels are in a similar form. So the main problem in this algorithm is to calculate this kind of integral. There are numerous articles that present certain methods of solving such integral, e.g. (Byrd and Friedmann, 1954) or (Prudnikov, Brychkov, Marichev, 1986). Additional difficulties may occur in connection with the transformation of coordinates, so the equations for transformations should be known. Since the planetocentric and planetographic coordinates are most commonly used in practice, the paper presents the graticules of these coordinate systems of a triaxial ellipsoid in designed map projections.

\section{Acknowledgments}

The research presented in this paper has been supported by statutory subsidies at the Faculty of Geodesy and Cartography of Warsaw University of Technology. No. 504/01826/1060/40.000XXX

\section{References}

Bektaş, S. (2014). Shortest distance from a point to triaxial ellipsoid. International Journal of Engineering and Applied Sciences, 4 (1), 22-26.

Bektaş, S. (2015). Geodetic Computations on Triaxial Ellipsoid. International Journal of Mining Science (IJMS), 1 (1), 25-34.

Byrd, P.F., Friedmann, M.D. (1954). Handbook of elliptic integrals for engineers and phisicists. SpringerVerlag, Berlin-Gottingen-Heidelberg.

Bugaevsky, L.M. (1987). K voprosu o poluchenii izometricheskikh koordinat i ravnougol'noy tsilindricheskoy proyektsii trekhosnogo ellipsoida. Izvestiya Vysshikh Uchebnykh Zavedeniy. Geodeziya i Aerofotosyemka, 4, 79-90.

Bugaevsky, L.M. (1991). Izometricheskiye koordinaty, ravnougol'noy tsilindricheskoy, konicheskoy i azimutal'noy proyektsii trekhosnogo ellipsoida. Izvestiya Vysshikh Uchebnykh Zavedeniy. Geodeziya i Aerofotosyemka, 3,144-152.

Bugaevsky, L.M. (1999). Teoria kartograficheskikh proyektsiy riegularnykh povierkhnostiey. Zlatoust.

Fleis, M. E., Nyrtsov, M. V., Borisov, M. M. (2013): Cylindrical Projection Conformality of Triaxial Ellipsoid. Doklady Earth Sciences, 45191, 787-789. DOI: 10.1134/S1028334X13070234 
Ligas, M. (2012). Cartesian to geodetic coordinates conversion on a triaxial ellipsoid. J. Geod., 86, 249-256. DOI:10.1007/s00190-011-0514-7

Nyrtsov, M. V., Bugaevsky, L. M. and Stooke, P. J. (2007). The multiple axis ellipsoids as reference surfaces for mapping of small celestial bodies. Proceedings of the International Cartographic Conference. Moscow, Russia, 4-10 August 2007.

Nyrtsov, M.V., Fleis, M.E. and Borisov, M. M. (2012). Kartografirovaniye asteroida 433 Eros v ravnopromezhutochnykh vdol' meridianov tsilindricheskoy i azimutal'noy proyektsiyakh trekhosnogo ellipsoida. Izvestiya Vysshikh Uchebnykh Zavedeniy. Geodeziya i Aerofotosyemka, 1, 54-61.

Nyrtsov, M. V., Fleis, M. E., Borisov, M. M. and Stooke, P. J. (2013). Equal-area projections of the triaxial ellipsoid: first time derivation and implementation of cylindrical and azimuthal projections for small solar system bodies. The Cartographic Journal, 52(2), 114-124. DOI:10.1080/00087041 .2015 .1119471

Nyrtsov, M. V., Fleis, M. E., Borisov, M. M. and Stooke, P. J. (2014). Jacobi Conformal Projection of the Triaxial Ellipsoid: New Projection for Mapping of Small Celestial Bodies. In. M. Buchroithner et al. (eds.). Cartography from Pole to Pole, Lecture Notes in Geoinformation and Cartography. DOI: 10.1007/978-3-642-32618-9_17, Springer-Verlag Berlin Heidelberg.

Prudnikov, A.P., Brychkov, Y.A. and Marichev, O.I. (1986). Integrals and series. Elementary functions, vol. 1. Gordon and Breach, New York.

Snyder, J.P., (1985). Conformal mapping of the triaxial ellipsoid. Survey Review, 28(217), 130-148. geocnt.geonet.ru/en/3_axial, accessed 03.09.2017

sbn.psi.edu/pds/archive/sat.html accessed 03.09.2017

planetarynames.wr.usgs.gov accessed 03.09.2017 